\title{
Um mapeamento de estudos de cores frente ao Modelo SENS|ORG|INT de percepção visual de modo a identificar princípios cromáticos que tendem a ser generalizáveis aos seres humanos
}

\author{
Mapping colour studies regarding visual perception Model SENS/OR/INT in order to \\ identify chromatic principles which tend to be generalized to human beings
}

Paula Csillag

Palavras-chave: cor, percepção visual, design

\begin{abstract}
Este artigo discute teorias existentes sobre cor e como estas relacionam-se com o modelo de percepção visual SENS|ORG|INT, que pode ser usado no design. O intuito principal deste modelo é diferenciar conceitos e princípios de design e linguagem visual que tendem a ser generalizáveis a todos os seres humanos com visão normal, daqueles conceitos e princípios que não tendem. Estes últimos são culturais, aprendidos ou interpretados de alguma forma pelo observador. Além deste, há um outro motivo para a elaboração deste modelo que é a intenção de unificar a interdisciplinaridade do estudo da percepção. Encontram-se estudos sobre percepção visual advindos de diferentes disciplinas como, psicologia, neurologia, design e artes. Com esta estrutura, agora aplicada a cor, profissionais que lidam com imagética podem diferenciar conceitos relacionados à sintaxe da linguagem cromática válidos como "leis", daqueles conceitos que não podem ser generalizados a todos os seres humanos.
\end{abstract}

Key-words: colour, visual perception, design

This paper discusses existing theories on colour and how they relate to visual perception model SENS/ORG/INT, which may be used in design. The main goal of this model is to differentiate visual literacy and design concepts and principles that tend to be valid to all human beings with normal eyesight, from those concepts and principles that don't. The latter are cultural, learned or otherwise interpreted in a way by the observer. Apart from this, there is another reason for the elaboration of this model, which is to unite different fields for the study of perception. There are studies of visual perception from different fields, such as, psychology, neuroscience, design, and arts. With such a framework, now applied to colour, professionals dealing with images, can differentiate concepts related to chromatic visual literacy valid as "laws" from those concepts that cannot be generalized to all human beings.

\section{Introdução}

O objetivo deste artigo é mapear analiticamente teorias cromáticas à luz do Modelo SENS|ORG|INT de Percepção Visual. O intuito principal deste modelo é diferenciar conceitos e princípios de design e linguagem visual que tendem a ser generalizáveis a todos os seres humanos com visão normal, daqueles conceitos e princípios que não tendem, uma vez que são culturais, aprendidos ou interpretados de alguma forma pelo observador. Além deste, há um outro motivo para a elaboração deste modelo que é a intenção de unificar a interdisciplinaridade do estudo da percepção. Encontram-se estudos sobre percepção visual advindos de diferentes disciplinas como, psicologia, neurologia, design e artes. Mesmo dentro da mesma disciplina, como por exemplo, a psicologia, há diferentes abordagens para explicar a percepção, como as abordagens analíticas e sintéticas. O presente modelo unifica não somente estas diferentes disciplinas como também diferentes abordagens, com o intuito de poder compreender melhor a análise e a produção de imagens.

Este modelo foi criado pela autora e premiado pelo 2008 Book of Selected Readings da Organização International Visual Literacy Association (CSILLAG, 2008), com o Prêmio Editor's Choice Award. Ao articular este modelo com teorias cromáticas, pode-se facilitar a identificação de parâmetros para criação e análise de imagens, no que diz respeito à eficácia de sua comunicação com cores. 


\section{Bases científicas para elaboração do modelo SENS|ORG|INT de percepção visual}

Vemos estudos e reflexões sobre percepção vindos de filósofos, místicos, psicólogos, neurologistas, artistas, designers, entre outros. Analisando cuidadosamente esta ampla gama de vertentes sobre a percepção visual, aqui foram eleitas as disciplinas de psicologia e neurologia para embasar a elaboração do modelo de percepção visual SENS|ORG|INT. Isto pois ambas disciplinas proporcionam explicações com fundamentos científicos para os elementos de percepção visual fisiológicos iniciais.

\section{Percepção visual na psicologia}

No século XIX, a percepção era estudada como uma estampagem "passiva" realizada por estímulos exteriores na retina. Em seguida, o córtex visual, que é a zona occipital do córtex cerebral, receberia os estímulos gerados na retina resultando em uma imagem idêntica (isomórfica) ao estímulo primário.

A psicologia moderna refuta esta noção e apresenta a percepção como um processo ativo que envolve a busca por informações correspondentes, a diferenciação de aspectos essenciais de uma imagem, a comparação destes aspectos entre si, a formulação de hipóteses apropriadas e a comparação destas hipóteses com os dados originais (Bruner, 1957; LEONTIEV, 1959; LURIA, 1981; VYGOTSKY, 1956; 1960; ZAPOROZHETS, 1967; 1968). Imagens familiares e não familiares podem ser diferenciadas por caminhos de percepção mais longos ou mais contraídos (LURIA, 1981).

Telford (1970) diferenciou sensação de percepção no sentido de que a primeira consiste de uma simples consciência das dimensões da experiência, enquanto percepção implica na sensação e nos significados que são atribuídos à experiência. Assim, para este autor, os determinantes da percepção são: contexto, constância, distância, perspectiva, interposição, luminosidade, posição, direção, acomodação, convergência, motivação, emoção e personalidade.

Alguns autores afirmam que percepção é um processo que medeia ou intervém entre a sensação e o comportamento. É iniciado pela sensação, mas não inteiramente determinado por ela (WHITTAKER, 1977). Os princípios da percepção e a maneira como processamos as informações ao nosso redor funcionam de uma maneira integrada (KRECH \& CRUTCHFIELD, 1976). Para Bruce, Green \& Georgeson (2003) a percepção é a habilidade de um ser de detectar estruturas e eventos do seu ambiente.

As teorias de percepção tendem a enfatizar o papel ou dos dados sensoriais ou do conhecimento no processo. Alguns teóricos adotaram uma abordagem conhecida como "datadriven" (orientada por dados), "bottom-up" (de baixo para cima), ou sintética, de acordo com a qual a percepção é direta. Nesta abordagem, os dados visuais são imediatamente estruturados no sistema visual antes de qualquer seletividade por parte do observador, abordagem esta adotada por Hering (1850), teorias da Gestalt e Gibson (1979). Outros teóricos adotaram uma abordagem conhecida como "top-down" (de cima para baixo), construtivista ou analítica, que enfatiza a importância do conhecimento prévio e hipóteses, abordagem defendida por Berkeley (1709), Helmoholtz (1925) e Bruce, Green \& Georgeson (2003).

\section{Percepção visual na neurociência}

Sabe-se que o cérebro é composto por regiões que, de maneira geral, ligam-se a diferentes órgãos sensoriais. Assim, no caso da visão humana, a região do cérebro principalmente responsável pelo processamento dos estímulos captados pelo olho corresponde ao córtex visual no lobo occipital. Para que o primeiro estágio da análise visual possa ocorrer no córtex visual, são necessários neurônios especializados (HUBEL \& WIESEL, 1963).

O cérebro humano está sendo estudado em detalhes e uma maneira de organizar o estudo de diferentes funções do cérebro foi dividir as regiões especializadas em áreas mais específicas. Assim, o córtex visual consiste do córtex visual primário (também chamado de córtex estriado ou V1) e as áreas visuais do córtex extrastriado, contendo as áreas V2, V3, V4 e V5. Cada uma destas áreas têm suas especializações, e no caso da percepção de cor, identificou-se uma pequena área de células em cada lado do cérebro que pareciam primariamente especializadas nesta função, a área V4 (ZEKI, 2000). 
Damasio (1996) apresenta um diagrama (vide Figura 1) de conexões existentes entre os córtices visuais iniciais (V1, V2, V3, V4 e V5), e três estruturas suborticais visualmente relacionadas, que são, o Núcleo Geniculato Lateral (NGL); o Pulvinar (PUL) e o colículo superior (COL). O autor ratifica que a maioria dos componentes deste sistema está interligado por projeções de neurônios que são estabelecidas nos dois sentidos (setas). O Modelo SENS|ORG|INT, contempla este diagrama, entretanto, abrange outros aspectos e destina-se à aplicação na comunicação visual.

Figura 1: O Modelo SENS|ORG|INT Diagrama simplificado de algumas interconexões existentes entre os córtices visuais iniciais (V1, V2, V3, V4 e V5). Fonte: Damasio (1996:118)

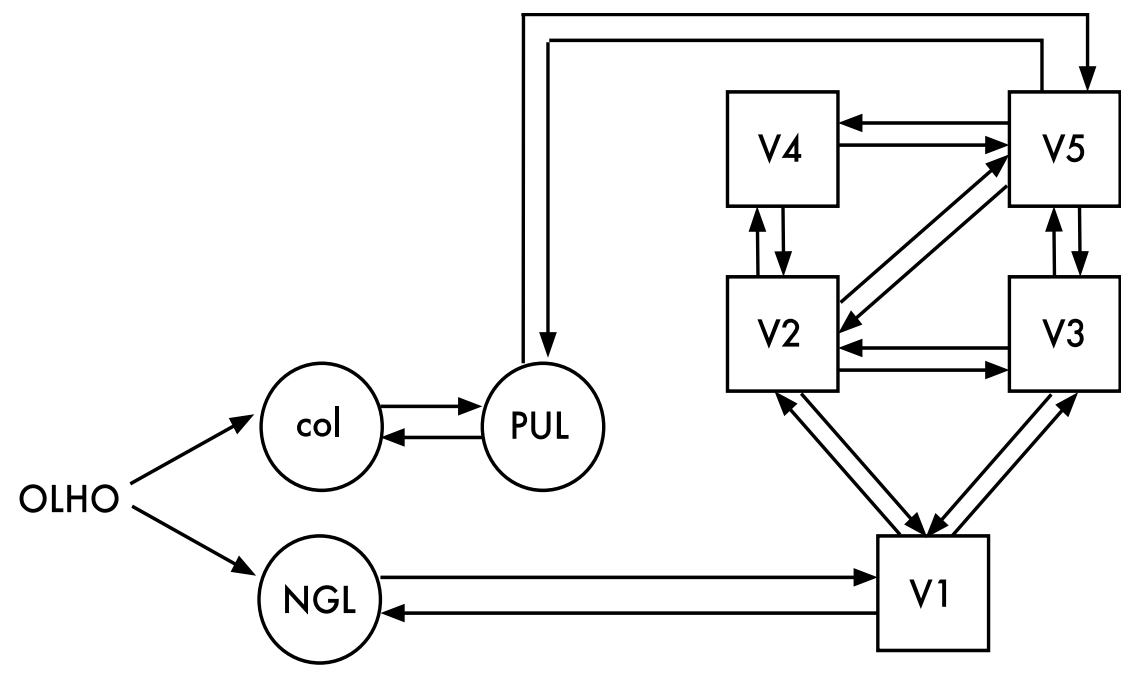

Recentes estudos da neurociência mapearam os percursos visuais (visual pathways) (CHALUPA \& WERNER, 2004; GATASS ET AL, 2001; KNOBLAUCH \& SHEVELL, 2004; PINNA \& SPILLMAN, 2001; SHIMOJO, KAMITANI \& NISHIDA, 2001; SPILLMAN \& LEVINE, 1971; ZEKI, 2000) e determinaram que a percepção ocorre por uma cascata neural, ativando áreas do cérebro que geralmente estão bem distantes entre si. Portanto, a percepção não ocorre por processos isolados no cérebro.

Ainda segundo Damasio (1996), o número e a complexidade de estruturas cerebrais e padrões de conexão que se encontram localizadas entre os setores de entrada e saída é enorme. Entre os principais setores sensoriais de entrada e os principais setores de saída do cérebro, encontram-se os córtices de associação, os gânglios basais, o tálamo, os córtices do sistema límbico e os núcleos límbicos, o tronco cerebral e o cerebelo. Ao todo, esse grande conjunto de sistemas, detém tanto o conhecimento inato como o adquirido sobre o corpo propriamente dito, sobre o mundo exterior e sobre o próprio cérebro, à medida em que esse interage com o corpo propriamente dito e com o mundo externo.

Os processos da Visão Primitiva considerados bottom-up pelos neurocientistas, que são os processos que não necessitam de conhecimento prévio e não são determinados por aprendizado ou experiência, são as percepções de movimento, profundidade, forma e cor. Cor pode até ser produzida experimentalmente por um estímulo magnético na área V4, propiciando a "visão" de anéis coloridos e halos, que foram denominados cromatofenos (SACKS, 2003). 


\section{O modelo SENS|ORG|INT e confronto de suas variáveis perante literatura de design e artes}

O Modelo SENS|ORG|INT foi elaborado de maneira a propiciar a compreensão da percepção visual para a prática em design e comunicação visual, baseado no estudo científico da percepção. O intuito principal do Modelo SENS|ORG|INT é diferenciar conceitos e princípios de design e linguagem visual que tendem a ser generalizáveis a todos os seres humanos com visão normal, daqueles conceitos e princípios que não tendem, uma vez que são culturais, aprendidos ou interpretados de alguma forma pelo observador. O Modelo SENS|ORG|INT encontra-se na Figura 2, abaixo e as variáveis intrínsecas ao modelo são: SENS, ORG e INT, respectivamente explicadas a seguir.

Figura 2: O Modelo SENS|ORG|INT

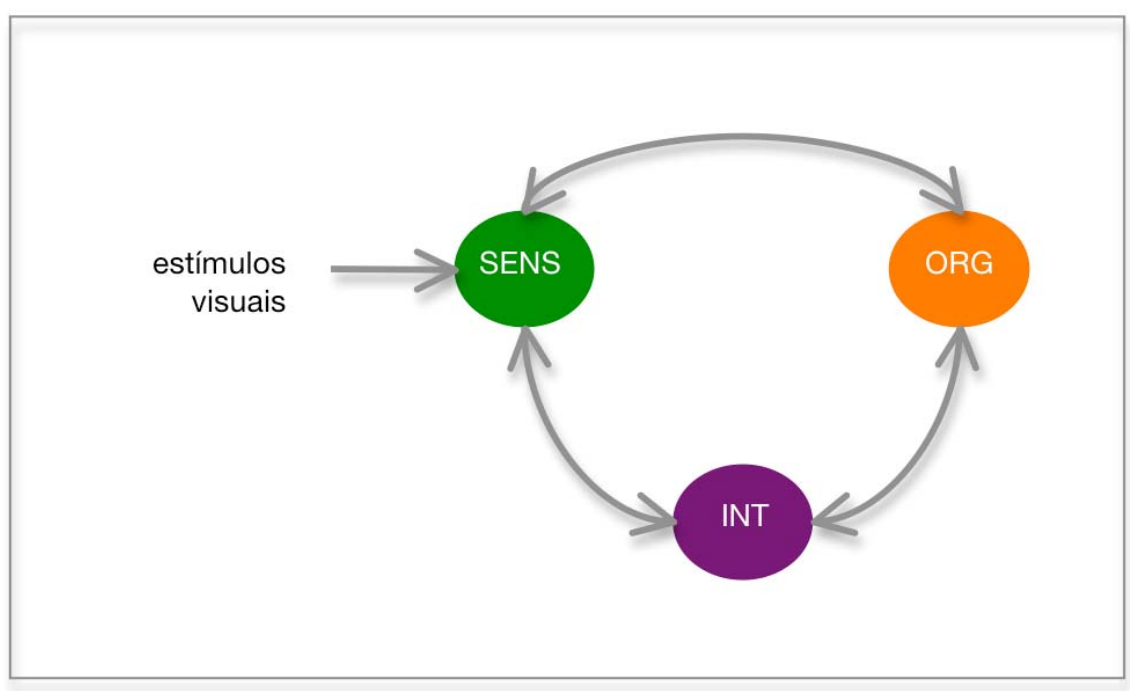

A variável SENS, está relacionada com o caminho da luz nos órgãos receptores visuais (os olhos) antes dela ser processada neuronalmente pela retina. Este aspecto da percepção é um fenômeno que ocorre apenas no olho, relacionando-se às informações visuais sensitivas. Note-se, que alguns autores nem consideram esta fase como "percepção", propriamente dita.

A variável ORG refere-se aos processamentos neuronais que começam na retina e incluem o chamado córtex visual primário na área V1 do córtex estriado. Esta variável está relacionada com os fenômenos da percepção visual que podem ser considerados "leis" em design e arte, assim como foram denominadas as leis da Gestalt. É predominantemente nesta etapa da percepção que ocorrem os fenômenos organizativos das imagens no cérebro.

A variável INT está relacionada com a elaboração de ORG a outras áreas do cérebro. É nesta fase da percepção que ocorrem as cascatas neurais, recebendo interferências de motivação, emoção, personalidade, cultura, conhecimento, entre outros fatores. Este aspecto da percepção propicia variações e interpretações pessoais das imagens que cada um elabora a partir de seu próprio repertório. Pensando-se topograficamente, não há uma localização exata no cérebro para INT, pois trata-se dos meandros mais complexos de interrelações e elaborações cerebrais, isto sem mencionar a plasticidade do cérebro, que permite compensações funcionais.

O Quadro 1 a seguir, apresenta um confronto das variáveis do modelo perante literatura de design e artes. A partir dos textos de cada autor, fez-se a classificação aproximada de cada conceito apresentado perante as variáveis SENS, ORG e INT (aproximada pois nenhum autor fez sua classificação pensando nas variáveis idênticas às do modelo). 
Quadro 1: confronto das variáveis SENS, ORG e INT do modelo perante literatura de design e artes Elaborado pela presente autora a partir das fontes citadas.

\begin{tabular}{|c|c|c|c|}
\hline AUTOR & SENS & ORG & INT \\
\hline MUNARI (1968) & Filtros Sensoriais & $\begin{array}{l}\text { Filtros psico fisiológicos; } \\
\text { Coerência formal; } \\
\text { Suporte para a mensagem visual }\end{array}$ & Filtros culturais \\
\hline GOMBRICH (1959) & Sensação visual & Ver & $\begin{array}{l}\text { llusão, } \\
\text { representação e } \\
\text { estilo }\end{array}$ \\
\hline WONG (1993) & & $\begin{array}{l}\text { Elementos conceituais, visuais e } \\
\text { relacionais }\end{array}$ & $\begin{array}{l}\text { Elementos } \\
\text { práticos }\end{array}$ \\
\hline SCOTT (1979) & & Fatores de atração & $\begin{array}{l}\text { Fatores de } \\
\text { atenção }\end{array}$ \\
\hline ITTEN (1973) & & Contrastes de cor & Timbre subjetivo \\
\hline ARNHEIM (1954) & & $\begin{array}{l}\text { Forças perceptivas; } \\
\text { Princípios subjacentes }\end{array}$ & $\begin{array}{l}\text { Elementos de } \\
\text { interesse } \\
\text { intrínseco }\end{array}$ \\
\hline KEPES (1944) & & Organização plástica & $\begin{array}{l}\text { Organização de } \\
\text { emoções }\end{array}$ \\
\hline DONDIS (1999) & & $\begin{array}{l}\text { Mensagem visual pura; } \\
\text { Sub estrutura; } \\
\text { Composição elementar abstrata } \\
\text { formada sintaticamente com os } \\
\text { elementos visuais básicos }\end{array}$ & $\begin{array}{l}\text { Imagem } \\
\text { comunicativa; } \\
\text { Simbolismo; } \\
\text { Representação; } \\
\text { Estilo; Função }\end{array}$ \\
\hline GREENBERG (1953) & & Formalismo & \\
\hline GUIMARÃES (2000) & $\begin{array}{l}\text { Códigos de } \\
\text { comunicação } \\
\text { hipolinguais, ou } \\
\text { primários }\end{array}$ & $\begin{array}{l}\text { Códigos de comunicação linguais } \\
\text { ou secundários }\end{array}$ & $\begin{array}{l}\text { Códigos de } \\
\text { comunicação } \\
\text { hiperlinguais ou } \\
\text { terciários }\end{array}$ \\
\hline
\end{tabular}

\section{Mapeamento de estudos cromáticos referentes à variável ORG do modelo SENS|ORG|INT}

Dos estudos cromáticos levantados, ressalta-se o valor dos contrastes cromáticos para os processos ORG. Isto pois enquadram-se na variável ORG, uma vez que tendem a propiciar o mesmo efeito perceptivo a seres humanos com visão normal, independentemente de cultura, aprendizado anterior, gosto ou variável subjetiva.

De longe, o contraste mais comentado pelos autores é o contraste simultâneo, devido à sua natureza intrigante e resultados perceptivos. Artistas como Delaunay (1885-1941) e Van Gogh (1853-1890) admitiam que realizaram obras a partir do conhecimento obtido com os estudos deste contraste. Delaunay foi particularmente influenciado por Chevreul (1987 [1854]). Em sua época, este autor, para estudar o fenômeno, desenvolveu diversas tapeçarias com o intuito de analisar as interações das cores. E, especificamente, para compreender o contraste simultâneo, o autor desenvolveu pranchas coloridas ilustrando como uma cor pode variar, dependendo da cor em sua adjacência.

Autores subsequentes que abordaram o mesmo contraste foram Albers (1974) e Itten (1973), Graves (1951), Evans (1948), Birren (1978, 1986), Beck (1972), Gerritsen (1976), Hichthier (1973), Kuppers (1975), Pedrosa (1995) e Sausmarez (1974). A propósito, Pedrosa (1995) tem o mérito de ter desenvolvido, ao longo de toda uma vida de dedicação a este estudo, pesquisa sobre o que denominou de "cor inexistente", fenômeno consequente do contraste simultâneo, e por isto tendo recebido um prêmio internacional "Prêmio Thomas Mann", na Alemanha.

Como o estudo da linguagem visual ainda é relativamente recente, as nomenclaturas utilizadas por diferentes autores nem sempre coincidem, mesmo discutindo a respeito do mesmo contraste. Por exemplo, referente ao contraste da Cor em Si, Pedrosa (1995, p. 174) faz referência ao que denominou "Contraste de Tons e Valores", também citado por Beck (1972), Gerritsen (1976), Hichthier (1973), Kuppers (1975). 
Conforme Itten (1973) apresenta, o contraste da cor em si, é aquele em que é utilizado o matiz puro adjacente com preto, produzindo assim matizes vibrantes. Neste contraste, o matiz puro comparado com fundo branco esmorece-o.

Segundo Itten (1973), o contraste de claro-escuro é importante para visualizar-se um efeito espacial. Isto é obtido mediante o grau do contraste. Com o fundo branco, o tom que mais avança é o mais escuro, enquanto que com o fundo preto, o tom que mais avança é o mais claro.

Um exemplo de aplicação deste contraste nas artes, pode ser vista na obra de um dos maiores mestres do chiaro-scuro, Rembrandt (1606-1669). O fundo escuro torna a obra um tanto quanto mais dramática. Victor Vasarely (1908-1997), sabiamente aplicando o conceito de espacialidade advinda do contraste de claro-escuro, obtém um efeito perceptivo de ilusão óptica. O observador é confundido com a volumetria do sólido ilustrado. Não se sabe se partes do sólido (cubos) vêm para frente ou vão para trás; se trata-se de convexidade ou concavidade.

O contraste de claro-escuro também foi abordado por Evans (1948), Birren (1978, 1986), Beck (1972), Gerritsen (1976), Wong (1997) E Sausmarez (1974), novamente com algumas variantes de nomenclaturas. Por exemplo, Wong (1997, p. 61) fala em graduações de valor, e importantemente, o autor comenta a respeito do efeito perceptivo na comunicação cromática deste contraste: "graduações de valor são eficazes para criar ilusões espaciais; valores baixos contra um fundo bem escuro somem na distância, mas altos valores parecem vir para frente". Já Sausmarez (1974) comenta sobre este contraste sem falar da cor do fundo, mas sim a respeito das distâncias tonais; quanto mais afastadas, mais espacial o efeito perceptivo.

Referente ao contraste de quente-frio explicado, os autores que melhor o descreveram foram Wong (1997) E Sausmarez (1974). Note-se aqui uma importante observação: encontrou-se a referência a cores quentes e cores frias em diversos outros autores também. Entretanto, estes não fizeram referência ao contraste de quente-frio, que é muito diferente de se observar as cores isoladas, como notado anteriormente. Wong (1997) E Sausmarez (1974), assim como Albers (1974) e Itten (1973), são dos poucos autores que comentam a respeito da relatividade do que seja considerado como quente ou frio, dependendo da adjacência cromática.

O contraste de complementar também foi amplamente abordado por Graves (1951), Evans (1948), Birren (1978, 1986), Beck (1972), Gerritsen (1976), Hichthier (1973), Kuppers (1975), Pedrosa (1995), Sausmarez (1974) E Wong (1997). Contraste muito adotado na comunicação visual, posto que propicia "design que capta o olho" (WONG, 1997, p. 77) devido à valorização máxima da vibração de cada matiz (Itten, 1973) e "máximo de vitalidade" (Sausmarez, 1974:93).

Dois contrastes menos comentados por autores são de saturação e extensão. O contraste de saturação, além de (Itten, 1973), foi comentado por Wong (1997), discutindo a respeito da maior suavidade obtida com sua utilização. O contraste de extensão, foi comentado por Pedrosa (1995) ao observar as mutações cromáticas variando-se a quantidade de cada cor usada e por Itten (1973) apresentando até proporções para que se obtenha um equilíbrio de luminosidade.

\section{Importância dos fenômenos ORG para a identificação de elementos que tendem a ser generalizáveis aos seres humanos}

Um argumento justificador da importância dos fenômenos ORG para a compreensão de princípios de comunicação visual que tendem a ser generalizáveis reside no fato de que INT pode ser determinada pelo espectro de possibilidades proposta por ORG. Para ilustrar isto, veja-se as figuras 3 e 4 . A figura 3 apresenta linhas em uma composição estática, enquanto que a figura 4 apresenta uma composição dinâmica, ambas elaboradas a partir de princípios clássicos de linguagem visual, segundo Kepes (1995 [1944]), Arnheim (1997 [1954],), Munari (2000 [1968]), Ostrower (1983), Scott (1979), Wong (1993) e Dondis (1999). Cada uma das duas composições pode ser interpretada a partir de conotações positivas ou negativas. Por exemplo, a composição estática (Fig. 3) poderia ser interpretada como tediosa (negativa) ou serena (positiva). A composição dinâmica (Fig. 4), poderia ser interpretada como confusa (negativa) ou alegre (positiva). 


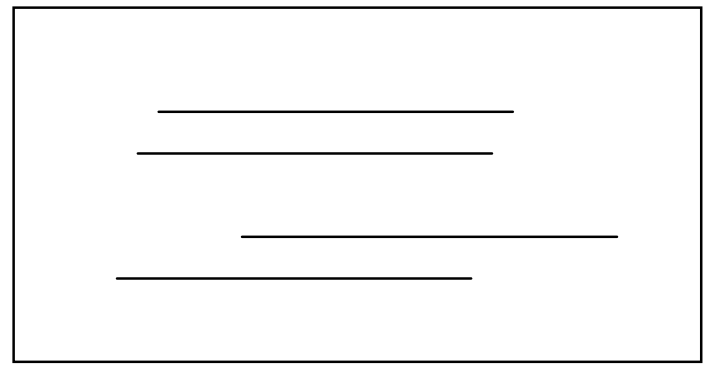

Figura 4: Esquema de Composição Dinâmica

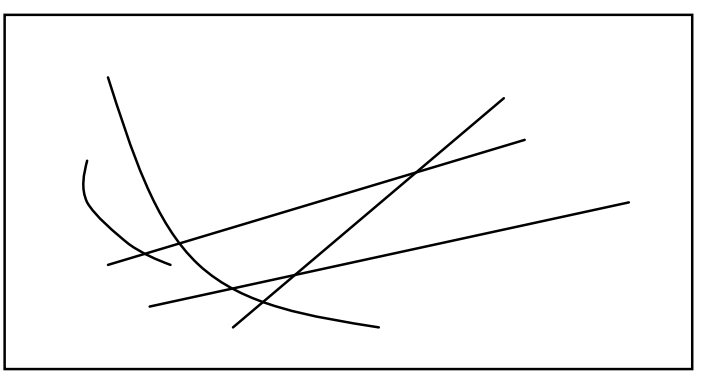

O caráter estático e o dinâmico de cada esquema compositivo estão relacionados com os processos organizativos do cérebro (ORG), e as interpretações das composições como serena ou alegre são interpretações subjetivas (INT). Agora, o aspecto importante desta discussão, é apontar que a composição da Figura 3 provavelmente não seria percebida como alegre e nem a composição da Figura 4 seria percebida como serena. Isto é relevante no sentido de evidenciar a importância de ORG na análise e produção de imagens.

\section{Considerações finais}

O objetivo deste artigo foi mapear analiticamente teorias cromáticas à luz do Modelo SENS|ORG|INT de Percepção Visual. Como vimos, o principal intuito deste modelo é diferenciar quais princípios de comunicação visual tendem a ser generalizáveis a todos os seres humanos com visão normal (ORG), de quais não tendem (INT). Na opinião da presente autora, princípios que enquadram-se como INT não devem ser apresentados como regras, e fornecem material justamente para a vontade criativa e de subversão de regras. Já os princípios generalizáveis (ORG) poderiam ser apresentados como "regras", apenas o termo talvez não fosse o mais indicado. Acredita-se aqui ser preferível usar o conceito de "natureza da visão" ao invés de regras, uma vez que o conceito de regra traz consigo a noção de que foi decidida por alguma instância ou instituição cultural. No caso de ORG, trata-se exclusivamente de como o olho em conjunto com o cérebro operam, por isso, o conceito de natureza da visão.

Portanto, vimos aqui a importância dos processos ORG para a comunicação visual. E também vimos que, da literatura sobre estudos cromáticos levantada, são principalmente os princípios relativos aos contrastes cromáticos que enquadram-se como processamento ORG da percepção visual. Assim, profissionais que lidam com design da informação podem aplicar na sua produção tais princípios, deixando livre o espaço para a criatividade nos processos INT de sua produção.

\section{Referências}

ALBERS, J. 1974. L'INTeractions de couleurs. Paris: Hachete (Edição Americana).

ARNHEIM, R. 1954. Arte e percepção visual. São Paulo: Ed. Pioneira.

BECK, J. 1972. Surface color perception. Ithaca: Cornell University Press.

BERKELEY, G. 1709. Berkeley selections. New York: Scribner's, 1929. 
BIRREN, F. 1978. Color and human response. New York: Van Nostrand Reinhold.

BIRREN, F. 1986. Color perception in art. Atglen: Schiffer Publishing.

BRUCE, V.,Green, P.; Georgeson, M. 2003. Visual perception. Hove: Psychology Press.

BRUNER, J. 1957. On Perceptual Readiness. Psychol. Rev., Vol. 64, No. 2.

CHALUPA, L.,Werner, J. (Eds.) 2004. The Visual Neurosciences. Cambridge: MIT Press.

CHEVREUL, M. E. 1987 [1854]. The principles of harmony and contrast of colors. West Chester: Schiffer.

CSILLAG, P. 2008. A Model of Visual Perception Useful for Designers and Artists. In: Griffin, R.; D'averignou, M. (Orgs.). Visual Literacy Beyond Frontiers: Information, Culture and Diversity. Loretto: St. Francis University Press, v., p. 11-20.

DAMASIO, A. 1996. O Erro de Descartes. São Paulo: Cia. das Letras.

DONDIS, D. 1999. Sintaxe da linguagem visual. São Paulo: Martins Fontes.

EVANS, R. 1948. An Introduction to color. New York: John Wiley \& Sons.

GATASS, R. et al. 2001. The brain decade in debate: VI. Sensory and motor maps dynamics and plasticity. Brazilian Journal of Medical and Biological Research. 34: 1497-1508.

GERRITSEN, F. 1976. Color: apariencia optica, medio de expresion artistica y fenomeno fisico.

Barcelona: Editorial Blume.

GIBSON, J. 1979. The ecological approach to visual perception. Boston: Houghton-Mifflin.

GOMBRICH, E. 1995. Arte e Ilusão. São Paulo: Martins Fontes.

GRAVES, M. 1951. The art of color and design. New York: McGraw Hill.

GREENBERG, C. 1961. Art And Culture: Critical Essays. New York.

GUIMARÃES, L. 2000. A Cor Como Informação. São Paulo: Annablume.

HELMOLTZ, H. 1925. Treatise on physiological optics: the perceptions of vision. Optical Society Of America, Ithaca.

HERING, E. 1850. Outlines of a Theory of the Light Sense. Cambridge.

HICHTHIER, A. 1973. Le cube des couleurs. Paris: Dessain el Tolra.

HUBEL, D.M.; WIESEL, T.N. 1963. Receptive Fields Of Cells In Striate Cortex Of Very Young, Visually Inexperienced Kittens. J. Neurophysiol., Vol. 26.

ITTEN, J. 1973. The art of color. New York: John Wiley \& Sons.

KEPES , G. 1944. Language of vision. New York: Dover Publications.

KNOBLAUCH, K.; SHEVELL, S. 2004. Color Appearance: The Visual Neurosciences. Cambridge: MIT Press.

KRECH, D., CRUTCHFIELD, R.1976. Elementos de psicologia. São Paulo: Ed. Pioneira, $2^{\circ}$ Vol.

KUPPERS, H. 1975. La couleur. Suisse, Office Du Livre.

LEONTIEV, A.N. 1959. Problems in mental development. Moscow: Izd. Akad.

LURIA, A.R. 1981. Fundamentos de neuropsicologia. Rio De Janeiro: Ed. Da Universidade De São Paulo.

MUNARI, B. 1968. Design e comunicação visual. Lisboa: Edições 70.

PEDROSA, I. 1995. Da cor à cor inexistente. Rio de Janeiro: Leo Christiano Ed.

PINNA, B., SPILLMAN, L. 2001. Surface Color From Boundaries: a new watercolor illusion. Vision Research, 41:2669-2676.

SACKS, O. 2003. Um Antropólogo em Marte. São Paulo: Cia. Das Letras.

SAUSMAREZ, M. 1974. Basic design: the dynamics of visual form. London: Studio Vista.

SCOTT, R. G. 1979. Fundamentos del diseño. Buenos Aires: Editorial Victor Leru. 
SHIMOJO S., KAMITANI Y., NISHIDA S. 2001. Afterimage of perceptually filled-in surface. Science. Aug 31;293(5535):1677-80.

SPILLMANN. L., LEVINE, J. 1971. Contrast enhancement in a Hermann grid with variable figureground ratio. Experimental Brain Research, 13: 547-559.

TELFORD, C. 1968. Psicologia. São Paulo: Cultrix.

VYGOTSKY, L. 1956. Selected psychological investigations. Moscow: Izd. Akad.

VYGOTSKY, L. 1960. Development of the higher mental functions. Moscow: Izd. Akad.

WHITTAKER, J. 1977. Psicologia. Rio De Janeiro: Ed. INTeramericana.

WONG, W. 1997. Principles of color design. New York: John Wiley \& Sons.

ZAPOROZHETS, A.V. 1967. Perception and action. Moscow: Prosv. Press.

ZEKI, S. 2000. The Architecture Of The Colour Centre In The Human Visual Brain: New Results And A Review. European Journal Of Neuroscience 12 (1), 172-193.

\section{Sobre a autora}

Paula Csillag. Dra. ESPM. É especialista e consultora em cores. Doutora e Mestre, é professora universitária do Curso de Graduação em Design da ESPM. Tem graduação em Artes Plásticas com Bacharelado em Pintura pela ECA-USP. Foi eleita em 2008 membro da Diretoria da International Visual Literacy Association (IVLA), onde em 2009 também foi eleita Vice Presidente. A partir de 2011 é membro do conselho científico e Vice Presidente da Associação Pró-Cor. paula@csillag.net 